\title{
Bei Älteren gelten andere Regeln als bei
} Jüngeren

\begin{abstract}
Bei älteren Diabetikern gibt es in der Therapie andere Prioritäten als bei jüngeren, erinnerte Prof. Werner Kleophas aus Düsseldorf. Der Erhalt einer hohen Lebensqualität steht im Fokus, eine strikte Blutzuckereinstellung ist weniger entscheidend. Die Wahl der Medikation sollte vor diesem Hintergrund erfolgen.
\end{abstract}

Das allgemeine Therapieziel bei Typ-2-Diabetikern $>65$ Jahre sind $\mathrm{HbA}_{1 \mathrm{c}}$-Werte von 7\%-8\% unter Vermeidung von Hypoglykämien, sagte Dr. Anke Bahrmann aus Heidelberg. Aber auch Komorbiditäten und Fitness der Patienten sollten berücksichtigt werden. So könnte bei fitten älteren Patienten ohne wesentliche körperliche Beeinträchtigungen ähnlich wie bei Jüngeren ein $\mathrm{HbA}_{1 \mathrm{c}}$-Wert von $6,5 \%$ angestrebt werden. Haben die Patienten allerdings bereits erhebliche Funktionseinbußen, sei der $\mathrm{HbA}_{1 \mathrm{c}}$-Wert sekundär, es gehe vor allem darum Hypo- und Hyperglykämien zu vermeiden.

Von großer Praxisrelevanz ist bei älteren Patienten die häufig bestehende Polymedikation. Ein Patient im Alter $>65$ Jahre nimmt im Schnitt 5 Arzneimittel ein, ein 80-Jähriger, bei dem im Mittel 3 Erkrankungen diagnostiziert worden sind, sogar 8-9, sagte Prof. Martin Wehling aus Mannheim. Leider gebe es fast keine Leitlinie zur Pharmakotherapie bei Älteren und nur wenige Daten zu den Auswirkungen einer Polymedikation. Besonders sorgfältig sollte auf die additive Wirkung von Medikamenten mit nephrotoxischer Wirkung geachtet werden, sagte Wehling. Zum Beispiel könne die Kombination von Metformin mit einem Coxib und einem ACE-Hemmer rasch zu einer Nierenschädigung führen. $\mathrm{Zu}$ beachten sei auch, dass neben vielen anderen Medikamenten (u.a. Trizyklika, Opiate, Benzodiazepine) auch orale Antidiabetika zu Verwirrtheit und einem erhöhten Sturzrisiko beitragen können. Hypoglykämien, die zu einer Klinikeinweisung führen, sind bei älteren Patienten weitaus am häufigsten, sagte Wehling. Deshalb sollten in dieser Altersgruppe vermehrt neuere Substanzen wie DPP-4-Hemmer eingesetzt

\begin{tabular}{ll} 
T1 Klassifizierung der Pharmaka zur Therapie bei Diabetes \\
mellitus Typ 2 nach Alterstauglichkeit \\
\hline Sulfonylharnstoffe, 3. Generation, niedrig dosiert (z.B. Glimepirid) & C \\
Sulfonylharnstoffe, 1. Generation (z.B. Glibenclamid) & D \\
Metformin & B \\
Acarbose & B \\
Glinide (als Vertreter Nateglinid) & C \\
PPAR-Gamma-Liganden (Pio-/ Rosiglitazon) & C/D \\
DPP-4-Hemmer & B (A?) \\
GLP-1-Analoga & B \\
Dapagliflozin & D \\
Insuline und Insulinanaloga & B \\
(von A=gut geeignet bis D=keinesfalls) Quelle: Wehling Diabeteskongress 2015, Berlin \\
\hline
\end{tabular}

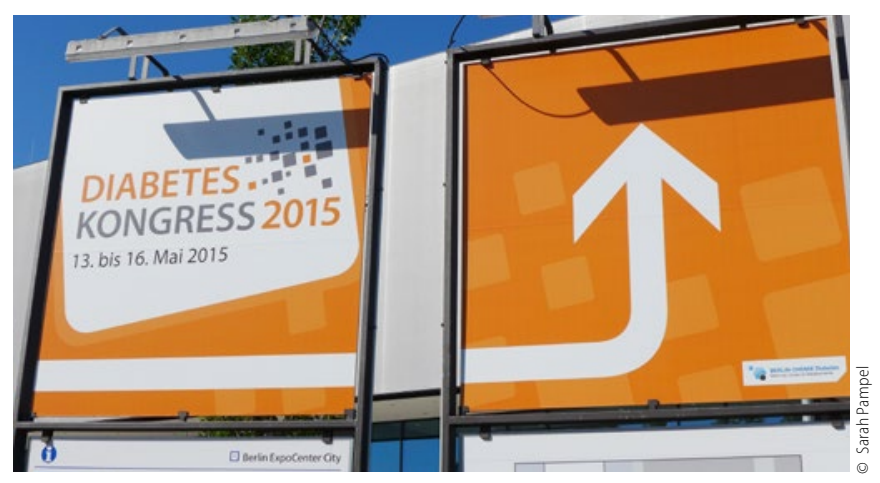

Da es in Deutschland immer mehr ältere Menschen mit Diabetes gibt, werden Probleme dieser Patientengruppe auch beim Diabeteskongress zunehmend thematisiert.

werden, die nur ein geringes Hypoglykämierisiko haben. „Sulfonylharnstoffe haben in der Altersmedizin nichts zu suchen", betonte Kleophas. Auch SGLT-2-Hemmer mit alterskritischen Nebenwirkungen wie Pilz- und Harnwegsinfektionen, so Wehling, sollten bei Älteren nicht unbedingt eingesetzt werden.

\section{Klassifizierung der Antidiabetika nach "Alterstauglichkeit"}

Der Pharmakologe empfahl eine Klassifizierung von Pharmaka zur Prophylaxe und Therapie des Diabetes mellitus nach Alterstauglichkeit in die Gruppen A (gut geeignet) bis D (nicht einsetzen) ( Tab. 1). Von Medikamenten aus der „kann“-Kategorie C, wie bei Antidiabetika die Glinide oder Sulfonylharnstoffe der 3. Generation, sollten nicht mehr als eins bis maximal 2 eingesetzt werden.

Laut der Heidelberger Diabetesstudie mit 77 Patienten, die in Pflegeheimen leben oder von Pflegediensten betreut werden, liegt die Inzidenz schwerer Hypoglykämien (Ereignis mit Fremdhilfe) bei knapp 8\% pro Patient und Jahr. Hypoglykämien korrelieren bei Diabetikern mit einem deutlich erhöhten kardiovaskulären Risiko, sagte Bahrmann, schwere Hyperglykämien mit erhöhter Krankenhausmortalität. Möglicherweise gebe es auch eine Verbindung zu einem erhöhten Risiko für kognitive Defizite und Demenz.

Neben der Blutzuckereinstellung mit nicht hypoglykämisierenden und nierensicheren oralen Antidiabetika hob Wehling die hohe Bedeutung einer guten Blutdruckeinstellung hervor und erinnerte an die UKPD-Studie. Hier war die Blutdrucksenkung in der Prävention kardiovaskulärer Komplikationen fünfmal so effektiv wie die des Blutzuckers. Ähnliches gelte für den Nutzen einer Lipidsenkung bei Fettstoffwechselstörungen.

Nicht vernachlässigt werden sollten auch bei älteren Patienten Lebensstilfaktoren wie mehr Bewegung, Gewichtsreduktion (in Maßen) und Rauchstopp. Dadurch könnten am meisten Pillen eingespart werden.

Roland Fath

Quellen: Diabeteskongress, 50. Jahrestagung der Deutschen Diabetes Gesellschaft (DDG), Berlin, Pressekonferenz und Symposium „Medikamente im Alter", 15.5.2015 